\title{
HCI-E $^{2}$ : HCI Engineering Education for developers, designers and more
}

\author{
Konrad Baumann ${ }^{1[0000-0001-7175-5927]}$, José Creissac \\ Campos $^{2[0000-0001-9163-580 X]}$, Alan Dix ${ }^{3[0000-0002-5242-7693]}$, Laurence \\ Nigay ${ }^{40000-0002-4854-626 X]}$, Philippe Palanque ${ }^{50000-0002-5381-971 X]}$, Jean \\ Vanderdonckt ${ }^{60000-0003-3275-3333]}$, Gerrit van der Veer ${ }^{7[0000-0001-5021-2042]}$, \\ and Benjamin Weyers ${ }^{8[0000-0003-4785-708 X]}$ \\ 1 FH Joanneum University of Applied Sciences, Austria \\ ${ }^{2}$ University of Minho \& HASLab/INESC TEC, Portugal \\ ${ }^{3}$ Computational Foundry, Swansea Universty, Wales, UK \\ ${ }^{4}$ University Grenoble Alpes, France \\ ${ }^{5}$ University of Toulouse, France \\ 6 Université catholique de Louvain, Belgium \\ 7 Vrije Universiteit Amsteredam, The Netherlands \\ 8 University of Trier, Germany
}

\begin{abstract}
This workshop aims at identifying, examining, structuring and sharing educational resources and approaches to support the process of teaching/learning Human-Computer Interaction (HCI) Engineering. The broadening of the range of available interaction technologies and their applications, many times in safety and mission critical areas, to novel and less understood application domains, brings the question of how to address this ever-changing nature in university curricula usually static. Beyond, as these technologies are taught in diverse curricula (ranging from Human Factors and psychology to hardcore computer science), we are interested in what the best approaches and best practices are to integrate HCI Engineering topics in the curricula of programs in software engineering, computer science, human-computer interaction, psychology, design, etc. The workshop is proposed on behalf of the IFIP Working Groups 2.7/13.4 on User Interface Engineering and 13.1 on Education in HCI and HCI Curricula.
\end{abstract}

Keywords: Human-Computer Interaction · Engineering · Education.

\section{Workshop Topics and Scope}

Engineering interactive systems is a multidisciplinary endeavour positioned at the intersection of Human-Computer Interaction (HCI), software engineering, usability engineering, interaction design, visual design and other disciplines. The field of Human-Computer Interaction Engineering (HCI-E) is concerned with providing methods, techniques, and tools for the systematic and effective design, development, testing, evaluation, and deployment of interactive systems in a wide range of application domains. 
The aim of such contributions is threefold:

- Improve the process of designing, developing, and evaluating interactive systems;

- Improve the quality of the user interface of interactive systems, including usability and user experience properties and software properties (also known as external and internal properties, respectively [3]);

- Adapt these contributions to the specific requirements and needs of the various application domains.

In recent years, the range of interactive techniques and applications has broadened considerably and can be expected to grow even further in the future. While new interaction techniques offer the prospect of improving the usability and user experience of interactive systems, they pose new challenges, not only for methods and tools that can support their design, development, and evaluation in a systematic engineering-oriented manner, but also to the designers and developers that must use them. This is aggravated by the fact that they are increasingly being applied in safety and mission critical, novel and less understood application domains (e.g., wearable medical devices and AI-based systems).

The techniques, methods and tools mentioned above, as well as many other novel forms of interaction, involve aspects that need to be adequately addressed in the curricula of programs in software engineering, computer science and HCI $[1,5,4,2]$. This begs the question of how best to address these topics in those curricula, and what the best approaches to address them are. Particularly relevant in the current context are approaches that support the educational process in an online context. When considering education about HCI Engineering, we need to think about who is being educated as there is likely to be different curriculum scope and educational methods for different types of learners. There are two main distinctions that are likely to influence these methods:

Technical vs. non-technical - Computer science and similar students are likely to be the main consumers of detailed HCIE education. However, it is also important for those who are likely to have a more interface design or user research role to be able to appreciate the limits of technology and the potential impacts of architectural design choices.

Student vs. practitioner - It is likely that the primary interest of many participants will be university education. However, developers are often involved in lively online discussions about different frameworks, and even the use of monads in interactive JavaScript. Interaction Design Foundation courses attract tens of thousands of UX practitioners worldwide, evidencing the desire for on-the-job learning in both communities.

Participants may target one or other of these types of learners, have interests that cover several, or indeed may address other groups.

\section{Goals and Expected Outcomes}

The workshop aims at identifying, examining and structuring educational resources and approaches to support the teaching/learning of HCI Engineering. 
It aims to cover a range of areas from challenges related to novel forms of interaction to emerging themes stemming from new application domains. Another goal is to consider the variety of students' skills and experiences. For instance, how to incorporate and teach HCI engineering in computer science curricula or in UI/UX design curricula? How to teach HCI engineering to students with different skills (e.g., engineers, designers)? The goal is also to consider different lecturing modalities, ranging from on-site lectures, project-based pedagogy to online/remote lecturing.

The intended outcome of the workshop is a structured overview of educational resources. This shall be structured according to the topics identified in the roadmap. This overview will take the form of an online resource, built around a wiki-style system, which will be made available to the community. We expect that, through this resource, educational materials (e.g., from slides and reference materials to exercises and exams) will be made available. It is expected that the workshop will result in the definition of a first instance of this resource and that this work will be continued in follow-up workshops, as well as in the context of IFIP Working Group 2.7/13.4 on User Interface Engineering ${ }^{9}$, where external participants are welcome. Depending on the quality of the submissions and the workshop results, revised versions of the contributions will be published on an edited volume. Alternatively, we will produce a journal paper summarizing and consolidating the contributions, in the form of an HCI Engineering Education roadmap. These results shall serve as a basis for drafting a roadmap for a curriculum for the engineering of advanced interactive computing systems and for identifying quality lecturing modalities.

\section{Target Audience}

Achieving the workshop's goals means bringing together expertise both on the Engineering of HCI and on education. Hence, we will solicit contributions from the HCI-E related communities and we will be very interested to welcome members of the educational community, for a fruitful discussion. To do so we will dispatch the call to the usual channels including announcements in mailing lists, conferences, and personal contacts.

\section{Submission}

Position papers (6-10 pages in LNCS format) must report experiences related to HCI Engineering education. Submissions could report software engineering units including some aspects of HCI-E, curricula or teaching units dedicated to HCI-E, case studies/projects demonstrating aspects of HCI-E, evaluation of students' skills related to HCI-E, training non-technical and mixed students in HCI-E, training appropriate aspects of HCI-E to professionals/practitioners, a new teaching modality promising for teaching HCI-E, introducing HCI-E into

\footnotetext{
${ }^{9}$ http://ui-engineering.org
} 
existing curricula, etc. Authors could also provide in their submission a short summary of their experience in the field and their motivation to participate in this workshop.

Submissions will be processed via the workshop web pages that will be hosted at the IFIP WG 13.4 web site. Position papers will be reviewed by the organizers, and participants will be invited to attend the workshop based on review results. All selected contributions will appear in the workshop proceedings that will be either an edited volume or a submission to a journal.

\section{Organisers Background and Experience}

The workshop is proposed on behalf of IFIP Working Groups 2.7/13.4 on User Interface Engineering and 13.1 on Education in HCI and HCI Curricula, and intends to further work ongoing within the groups.

WG 2.7/13.4 aims at advancing the state of the art in all aspects of designing, developing, and evaluating interactive computing systems with a particular focus on principled methodological engineering approaches. The scope of investigation comprises, among others, methods and tools for modelling, prototyping, developing and evaluating user interfaces (UI), quality models for interactive systems, and new interface technologies suitable to improve user interaction.

The WG understands UI Engineering as the creation and application of scientific knowledge and systematic, structured design and development methods to predictably and reliably improve the consistency, usability, scalability, economy and dependability of practical problem solutions. UI Engineering addresses all aspects related to methods, processes, tools, technologies, and empirical studies involved in the invention, design and construction of interactive systems. The techniques addressed concern all types of applications, for example, business applications, social media, smart environments, medical devices, automotive and aeronautics applications, and others.

The identification and organisation of educational resources for the topic has raised interest within the group in recent meetings. This interest was strengthened by the current pandemic crisis that requires novel approaches to support the teaching and learning process.

WG 13.1 aims at improving HCI education at all levels of higher education, coordinate, at coordinating and uniting efforts to enhance the development of HCI curricula, at recommending fundamental structures for curricula and course materials and for their adaptation to various national educational systems, at advancing international recognition of qualifications in this field, and at promoting the teaching of HCI. Since it is interested to gather all resources which can be effectively used in such HCI curricula, it is particularly interested in collecting, analyzing, and comparing models, methods, and tools for supporting the development of interactive applications and their UIs.

Konrad Baumann is chair of IFIP WG 13.1 Education in HCI and HCI Curricula. As a professor at University of Applied Sciences FH Joanneum in Graz, Austria, his teaching and research interests focus on HCI-related topics like 
usability testing, user-centred design, user interface design, information design, user experience design, interaction design and evaluation of exhibitions. He has been supervising more than 100 theses. He is co-author of two books, an editorial board member of the Information Design Journal (IDJ) and served as a board member of the International Institute for Information Design (IIID). He received his $\mathrm{PhD}$ in 2004 at the Institute for Design and Assessment of Technology at TU Vienna. Before that, he also worked in industry as a product manager.

José Creissac Campos is the chair of IFIP WG 2.7/13.4 on User Interface Engineering. He is an associate professor at the informatics department of the University of Minho, and a senior researcher at HASLab/INESC TEC. José chairs the Steering Committee of the ACM SIGCHI Symposium on Engineering Interactive Computing Systems (EICS) and is a member of the Editorial Board of the ACM Proceedings in Human-Computer Interaction journal. He has served in several organizing committees, including several ACM SIGCHI EICS, IFIP TC13 INTERACT 2011 and Formal Methods Week 2019. He regularly serves on the Program Committees of EICS, INTERACT and IUI, among others.

Alan Dix is Director of the Computational Foundry at Swansea University. $\mathrm{He}$ is author of one of the principle textbooks in Human Computer Interaction as well as many other research publications and a recent book on Statistics for HCI. He was the general chair of HCI-Educators 2007 as well as several more recent workshops in the area, including a series of Covid-related virtual workshops on video in HCI education early in 2020. He has worked in a number of commercial roles in addition to his academic posts and contributes to courses at Interaction Design Foundation. Alan still designs and codes interactive systems.

Laurence Nigay is a full Professor (Exceptional class) in Computer Science at the University of Grenoble Alpes (UGA) and is also an elected senior member of the Academic Institute of France (IUF). She is the director of the Engineering Human-Computer Interaction (EHCI) research group of the Grenoble Informatics Laboratory (LIG), comprised of 11 faculty members and more than 20 non-permanent members. From 1998-2004, she was vice-chair of the IFIP working group 2.7/13.4. She has been regularly involved in the ACM SIGCHI Symposium on Engineering Interactive Computing Systems (EICS): reviewer since 2009, member of the Editorial Board, program co-chair at EICS 2015, doctoral consortium co-chair at EICS 2014 and 2012. From 2005 to 2019 she was the director of the Masters of Computer Engineering at the University of Grenoble.

Philippe Palanque is a professor in Computer Science at the University Toulouse 3 and is head of the ICS (Interactive Critical Systems) research group at IRIT. Since the early 90's his research focus is on interactive systems engineering proposing notations, methods and tools to integrate multiple properties such as usability, dependability, resilience and more recently user experience. These contributions have been developed together with industrial partners from various application domains such as civil aviation, air traffic management or satellite ground segments. He is currently one of the head of the Master of HCI in Toulouse (www.masterihm.fr) that was created in 2000. He was steering com- 
mittee chair of the CHI conference series at ACM SIGCHI, is a member of the CHI academy and chair of IFIP Technical Committee on HCI (TC13).

Jean Vanderdonckt is a full professor in Computer Science at Université catholique de Louvain where he leads the Louvain Interaction Lab, which is located midway between software engineering, HCI, and usability engineering. He is Vice-Chair of WG 13.1 Education in HCI and HCI curricula. He is coeditor-in-chief of Springer's HCI series of books and holder of the Francqui Chair in Computer Science 2020.

Gerrit van der Veer has been teaching at the Vrije Universiteit Amsterdam since 1961. He started in Cognitive Psychology, moved to Ergonomics, and into Computer Science, where he specialized in the design of interactive systems. He has been developing education in interaction design in many European countries, including Belgium, Germany, Spain, Italy, Romania, and the Netherlands, as well as in China. He is currently working for the Dutch Open University Department Human-Computer-Society, the Dutch University of Twente, Department of Human-Media Interaction, the University of Sassari (Italy) Faculty of Architecture and Design, the Luxun Academy of Fine Arts (China) Department of Multimedia and Animation, and the Maritime University of Dalian (China) Faculty of Computer Science. Gerrit has been President of ACM SIGCHI, the world leading international society for HCI, from 2009-2015.

Benjamin Weyers is currently Assistant Professor at University of Trier and Head of the Human-Computer Interaction Group. Before, he was PostDoc at the Virtual Reality and Immersive Visualization group at RWTH Aachen University. He received his PhD in 2011 at the University of Duisburg-Essen and joined the RWTH in 2013. He is interested in the research and development of interactive analysis methods for abstract and scientific data using immersive systems as well as the integration of VR and AR into the control of technical systems for the support of human users in semi-automated control scenarios. An integral element in this research is the application of modeling method as part of the engineering process. Therefore, Benjamin focuses on the development and use of formal methods for the description of interactive systems. He is member of IFIP WG 2.7/13.4 on User Interface Engineering.

\section{References}

1. ACM/IEEE-CS Joint Task Force on Computing Curricula: Computer science curricula 2013. Tech. rep., ACM Press and IEEE Computer Society Press (Dec 2013)

2. Churchill, E.F., Bowser, A., Preece, J.: Teaching and learning human-computer interaction: Past, present, and future. Interactions 20(2), 44-53 (Mar 2013)

3. Gram, C., Cockton, G. (eds.): Design Principles for Interactive Software. Chapman \& Hall (1996)

4. Hewett, T.T., Baecker, R., Card, S., Carey, T., Gasen, J., Mantei, M., Perlman, G., Strong, G., Verplank, W.: ACM SIGCHI Curricula for Human-Computer Interaction. Tech. rep., ACM, New York, NY, USA (1996)

5. The Joint Task Force on Computing Curricula: Software Engineering 2014: Curriculum guidelines for undergraduate degree programs in software engineering. Tech. rep., ACM \& EEE-Computer Society, New York, NY, USA (2015) 